\title{
Saúde digital e telemedicina aplicadas ao câncer colorretal: uma revisão
}

\section{Digital health and telemedicine applied to colorectal cancer: a review}

\author{
1 Joana Lustosa de Almeida lustosajoana@hotmail.com \\ 2 Eduardo Botelho Cabral \\ 3 Claudia Yamada Utagawa
}

\footnotetext{
1 Graduanda do curso de Medcina do Centro Universitário de Volta Redonda - UniFOA.

2 Graduando do curso de Medcina do Centro Universitário de Volta Redonda - UniFOA.

3 Doutora em Ciência pela Universidade Federal do Rio de Janeiro. Docente do Centro Universitário de Volta Redonda - UniFOA.
}

\section{Resumo}

O câncer colorretal é o $3^{\circ}$ mais comum entre os cânceres e um dos tipos de tumor com a mais alta taxa de mortalidade. $\mathrm{O}$ uso de tecnologias digitais (eHealth) pode ser útil por poder atuar em diversos aspectos do atendimento à doença. A telemedicina é um dos ramos da saúde digital que consiste no exercício da medicina através de tecnologias para diversos fins, como assistência de doenças e promoção de saúde. O objetivo deste trabalho foi compreender como a saúde digital e a telemedicina são empregadas na abordagem no câncer colorretal, verificando se há benefícios ou riscos para o paciente. Foi realizada uma revisão de literatura nas bases de dados PubMed, Scielo e Cochrane, no período de 2015 a 2020, em português e inglês, utilizando-se os descritores "colorectal cancer" and "telemedicine", "colorectal cancer" and "telehealth", "colorectal cancer" and "ehealth" and "colorectal cancer" and "telecare". A seleção dos artigos foi realizada de acordo com o PRISMA. Foram identificados 43 artigos dos quais 12 foram selecionados, sendo que 6 abordavam o acompanhamento do câncer colorretal após o tratamento e 6 tinham como foco o rastreamento da doença. A telemedicina, que poderia facilitar o apoio do médico ao doente, foi utilizada em quatro estudos. Considerando a grande incidência do CCR e suas altas taxas de mortalidade, é fundamental a introdução da telemedicina e telessaúde na vida dos pacientes. Por meio dela, pode-se alcançar populações cada vez maiores em curtos períodos de tempo.

\section{Palavras-chave:}

Telemedicina. eHealth. Câncer colorretal.

\begin{abstract}
Colorectal cancer is the 3rd most common cancer and one of the tumor types with the highest mortality rate. The use of digital technologies (eHealth) can be useful because it can act in different aspects of the disease's care. Telemedicine is one of the branches of digital health that consists of the practice of medicine through technologies for various purposes, such as disease care and health promotion. The objective of this study was to understand how digital health and telemedicine are used in the approach to colorectal cancer, verifying whether there are benefits or risks for the patient. A literature review was carried out in the PubMed, Scielo and Cochrane databases from 2015 to 2020 in Portuguese and English using the descriptors "colorectal cancer" and "telemedicine", "colorectal cancer" and "telehealth", "colorectal cancer" and "ehealth" and "colorectal cancer" and "telecare". The selection of articles was carried out according to PRISMA. A total of 43 articles were identified, of which 12 were selected, 6 of which addressed the follow-up of colorectal cancer after treatment and 6 sought to focus on tracking the disease. Telemedicine, which could facilitate physician support for the patient, has been used in four studies. Considering the large incidence of CCR and its high mortality rates, it is essential the introduction of telemedicine and telehealth in patients' lives. Through it it is possible to achieve population that gets bigger in short periods of time.
\end{abstract}

\section{Keywords:}

Telemedicine. eHealth. Colorectal cancer.

Como você deve citar?

ALMEIDA, Joana Lustosa de; CABRAL, Eduardo Botelho; UTAGAWA, Claudia Yamada. Saúde digital e telemedicina aplicadas ao câncer colorretal: uma revisão. Cadernos UniFOA, Volta Redonda (RJ), v. 16, n. 47, p. 147 - 155, dez, 2021. 
O câncer é a segunda causa de morte em países desenvolvidos e a terceira em países menos desenvolvidos, sendo o câncer colorretal (CCR) o terceiro mais comum entre os cânceres (BERKEL et al., 2018) e um dos tipos de neoplasia com as mais altas taxas de mortalidade. A taxa de sobrevivência de CCR, em cinco anos, é de 13,5\% (OVERMAN et al., 2017). A estimativa de casos de CCR, para cada ano do triênio de 2020-2022, no Brasil, é de 20.520 casos em homens e 20.470 casos em mulheres. Esses valores correspondem a um risco estimado de 19,63 casos novos a cada 100 mil homens e 19,03, para cada 100 mil mulheres (BRASIL, 2019).

Através do rastreio e acompanhamento clínico, pode-se detectar e remover lesões pré-cancerosas precocemente, impedindo o desenvolvimento do CCR, sendo, portanto, uma doença evitável. Mesmo que a doença já esteja instalada, os pacientes ainda podem se beneficiar do tratamento precoce, que é mais efetivo (PRESTON et al., 2018).

Um dos desafios é como atender a demanda crescente de pacientes, uma vez que o envelhecimento da população mundial acarreta o aumento de doenças crônicas, incluindo o câncer (LEWIS; RAY; LIAW, 2016). $O$ uso de tecnologias digitais poderia auxiliar no manejo, na educação e na gestão desses pacientes. Essa tecnologia, chamada genericamente de saúde digital (eHealth, em inglês) pode incluir o uso de dispositivos móveis e tecnologias wireless; sistemas de informação em saúde, como prontuários eletrônicos; a telemedicina e a educação a distância. Para a Organização Mundial da Saúde (OMS, 2005), a saúde digital consiste no uso seguro e custo-efetivo de tecnologias de informação e comunicação para apoiar a saúde e campos relacionados à saúde.

A telemedicina, portanto, é um dos ramos da saúde digital e consiste no exercício da medicina através de tecnologias para diversos fins, podendo ser citados: assistência, educação, pesquisa, prevenção de doenças e lesões e promoção de saúde. Em suma, a telemedicina oferta serviços de saúde por meio de telecomunicação, havendo troca de informações entre médico e paciente, sendo exemplos os serviços de consulta e diagnóstico (CONSELHO FEDERAL DE MEDICINA, 2019).

Desse modo, a implantação da telemedicina na vida do paciente resulta em inúmeros benefícios, como, por exemplo, melhorias no bem-estar emocional e funcional, enfrentamento, participação nas decisões e uso efetivo dos serviços de saúde (HAN et al., 2017).

Até o presente momento, não foram encontrados, na literatura, estudos que revisem a aplicabilidade da saúde digital e da telemedicina para auxiliar pacientes com CCR, seja no rastreio, tratamento, monitoração ou reabilitação.

Nesse contexto, o objetivo do presente artigo consiste em compreender como a saúde digital e, em especial, a telemedicina, é empregada na abordagem do CCR, verificando se há benefícios ou riscos para o paciente. Além disso, espera-se estabelecer os tipos de tecnologias de saúde digital utilizadas no CCR e sua aplicabilidade. 


\section{METODOLOGIA}

Foi realizada uma revisão de literatura nas bases de dados científicos PubMed, Scielo e Cochrane, contemplando os artigos publicados no período de 2015 a 2020, em português e inglês. A revisão foi de caráter qualitativo e descritivo.

Para a busca bibliográfica, foram utilizadas as combinações de descritores em inglês: "colorectal cancer" and "telemedicine", "colorectal cancer" and "telehealth", "colorectal cancer" and "ehealth" and "colorectal cancer" and "telecare". A seleção dos artigos foi realizada de acordo com o PRISMA (Preferred Reporting Items for Systematic Reviews and Meta-Analyses).

\section{RESULTADOS E DISCUSSÃO}

Foram identificados 43 artigos nas plataformas PubMed, Cochrane e Scielo, por meio dos descritores definidos na metodologia. Após a exclusão dos artigos duplicados e da avaliação dos resumos, foram elegíveis 12 artigos. Dos artigos selecionados, seis abordavam o acompanhamento do paciente com CCR após o tratamento e seis tinham como foco a triagem para a busca de possíveis novos pacientes e diagnóstico precoce da doença.

Em relação ao acompanhamento do paciente com CCR, todos os seis trabalhos utilizaram aplicativos, softwares ou sítios eletrônicos para tal finalidade. 0 Quadro 1 relaciona e resume os recursos tecnológicos, os objetivos e as estratégias utilizadas pelos estudos e seus principais resultados. 
Quadro 1. Estudos sobre o uso de recursos em saúde digital no acompanhamento de pacientes com CCR e resultados

\begin{tabular}{|c|c|c|c|c|c|c|c|}
\hline Autor e ano & $\begin{array}{c}\text { Recurso } \\
\text { tecnológico }\end{array}$ & Estratégia & Objetivo & $\begin{array}{l}\text { Tipo de } \\
\text { estudo }\end{array}$ & $\begin{array}{l}\text { Tempo de } \\
\text { estudo }\end{array}$ & $\begin{array}{l}\text { Número total de } \\
\text { participantes } \\
\text { (Intervenção/ } \\
\text { Controle) }\end{array}$ & Resultados \\
\hline $\begin{array}{l}\text { Bakker et al. } \\
\text { (2019) }\end{array}$ & Ikherstel & $\begin{array}{l}\text { Sítio eletrônico, } \\
\text { aplicativo móvel, } \\
\text { rastreador de } \\
\text { atividades e } \\
\text { consulta eletrônica } \\
\text { (eConsult) }\end{array}$ & $\begin{array}{l}\text { Medir o alcance } \\
\text { dos componentes } \\
\text { e dose do } \\
\text { medicamento, dose } \\
\text { recebida, fidelidade } \\
\text { e atitudes dos } \\
\text { participantes. }\end{array}$ & ECR & 3 meses & $151(73 / 71)$ & $\begin{array}{l}\text { Eficaz na } \\
\text { recuperação dos } \\
\text { pacientes após a } \\
\text { cirurgia colorretal. }\end{array}$ \\
\hline $\begin{array}{l}\text { Hout et al. } \\
\text { (2020) }\end{array}$ & Oncokompas & $\begin{array}{l}\text { Aplicativo para } \\
\text { suporte aos } \\
\text { pacientes através do } \\
\text { autogerên-cimento, } \\
\text { monitorando a } \\
\text { qualidade de vida } \\
\text { relacionada à saúde } \\
\text { (QVRS) e sintomas } \\
\text { genéricos de câncer } \\
\text { e específicos de } \\
\text { tumor. }\end{array}$ & $\begin{array}{l}\text { Avaliar questões } \\
\text { físicas, } \\
\text { psicológicas, } \\
\text { sociais, existência } \\
\text { ais, estilo de vida e } \\
\text { tópicos específicos } \\
\text { do tumor após o } \\
\text { tratamento dos } \\
\text { pacientes a fim } \\
\text { de reduzir a carga } \\
\text { de sintomas } \\
\text { e melhorar a } \\
\text { qualidade de vida. }\end{array}$ & $\begin{array}{l}\text { ECR não } \\
\text { cego }\end{array}$ & 6 meses & $625(320 / 305)$ & $\begin{array}{l}\text { Não obteve } \\
\text { melhora em } \\
\text { relação ao conheci } \\
\text { mento, habilidades } \\
\text { e confiança para } \\
\text { autocuidado em } \\
\text { sobreviven- tes de } \\
\text { câncer. }\end{array}$ \\
\hline $\begin{array}{l}\text { Mayer et al. } \\
\text { (2017) }\end{array}$ & SurvivorCHESS & $\begin{array}{l}\text { Aplicativo com } \\
\text { informações sobre } \\
\text { saúde e rastreio de } \\
\text { comportamentos de } \\
\text { saúde. }\end{array}$ & $\begin{array}{l}\text { Ajudar os } \\
\text { participantes a } \\
\text { aumentar seus } \\
\text { níveis de atividade } \\
\text { física diária. }\end{array}$ & ECR & 9 meses & $284(144 / 140)$ & $\begin{array}{l}\text { Eficaz em relação } \\
\text { ao aumento da } \\
\text { atividade física } \\
\text { em ambos os } \\
\text { grupos, não sendo } \\
\text { significativamente } \\
\text { diferente entre } \\
\text { eles. Porém, esse } \\
\text { resultado não foi } \\
\text { sustentado por } \\
3 \text { meses após o } \\
\text { término do estudo. }\end{array}$ \\
\hline $\begin{array}{l}\text { Bednarski et } \\
\text { al. (2019) }\end{array}$ & TeleRecovery & $\begin{array}{l}\text { Programa de } \\
\text { telemedicina }\end{array}$ & $\begin{array}{l}\text { Obter alta precoce } \\
\text { para os usuários do } \\
\text { programa. }\end{array}$ & ECR & 30 dias & $30(14 / 16)$ & $\begin{array}{l}\text { Eficaz para alta } \\
\text { precoce dos } \\
\text { pacientes no pós- } \\
\text { operatório de CCR. }\end{array}$ \\
\hline $\begin{array}{l}\text { Golsteijn } \\
\text { et al. (2018) }\end{array}$ & OncoActive & $\begin{array}{l}\text { Sítio eletrônico para } \\
\text { computador }\end{array}$ & $\begin{array}{l}\text { Aumentar a } \\
\text { conscientização, } \\
\text { iniciação e } \\
\text { manutenção da } \\
\text { atividade física } \\
\text { em pacientes e } \\
\text { sobreviventes com } \\
\text { câncer de próstata } \\
\text { e colorretal. }\end{array}$ & ECR & 12 meses & $478(249 / 229)$ & $\begin{array}{l}\text { Eficaz no aumento } \\
\text { da atividade física } \\
\text { em pacientes com } \\
\text { CCR de próstata } \\
\text { e sobreviven- tes, } \\
\text { durante e após } \\
\text { o tratamento do } \\
\text { câncer primário. }\end{array}$ \\
\hline $\begin{array}{l}\text { Eldeib et al. } \\
\text { (2018) }\end{array}$ & Telefone & $\begin{array}{l}\text { Telefonemas } \\
\text { semanais }\end{array}$ & $\begin{array}{l}\text { Acompanhar, } \\
\text { avaliar os } \\
\text { efeitos adversos } \\
\text { esperados e } \\
\text { recomendar } \\
\text { estratégias } \\
\text { adequadas para } \\
\text { tratamen- tos, } \\
\text { principal- mente a } \\
\text { capecitabina oral. }\end{array}$ & ECR & 5-6 meses & $82(44 / 38)$ & $\begin{array}{l}\text { Resultados } \\
\text { iniciais positivos, } \\
\text { porém não foram } \\
\text { significantes } \\
\text { devido ao pequeno } \\
\text { tamanho da } \\
\text { amostra. }\end{array}$ \\
\hline
\end{tabular}

ECR: Ensaio clínico randomizado

Fonte: Autores (2020) 
Verificou-se, portanto, que, nos estudos relacionados ao acompanhamento após o tratamento do CCR, um utilizou sítio eletrônico e aplicativo (BAKKER et al., 2019); um utilizou sítio eletrônico (GOLSTEIJN et al., 2018); três, aplicativo e/ou programas eletrônicos (MAYER et al., 2017; BEDNARSKI et al., 2019; HOUT et al., 2020); e um, telefonema (ELDEIB et al., 2019). Todos os artigos foram eficazes (MAYER et al., 2017; GOLSTEIJN et al., 2018; BAKKER et al., 2019; BEDNARSKI et al., 2019; ELDEIB et al., 2019; HOUT et al., 2020), contudo, um dos artigos, apesar de resultados positivos, o tamanho da amostra era pequeno para avaliar a significância dos achados (ELDEIB et al., 2019).

Sobre o emprego da telemedicina, apenas três trabalhos utilizaram esse recurso (BAKKER et al., 2019; BEDNARSKI et al., 2019; ELDEIB et al., 2019). No TeleRecovery, ao receberem alta, os pacientes eram agendados por meio do aplicativo para uma teleconsulta, realizada por videoconferência e mensagens instantâneas através de um iPad. Esse estudo demonstra a viabilidade de abordagens de curta duração com alta no dia seguinte após ressecção colorretal, porém ainda são necessários outros estudos para a confirmação da aplicação e relação custo-benefício (BEDNARSKI et al., 2019). Em um dos estudos, as consultas eram via telefone e focavam principalmente nos efeitos adversos de uma droga específica (capecitabina oral), porém o tamanho da amostra prejudicou a análise dos dados (ELDEIB et al., 2019).

Em relação à triagem de novos pacientes, os seis trabalhos apresentaram abordagens e objetivos diversos, como apresentado no Quadro 2, que resume também os principais resultados. 
Quadro 2. Estudos sobre o uso de recursos em saúde digital na triagem de pacientes com CCR e resultados

\begin{tabular}{|c|c|c|c|c|c|c|c|}
\hline $\begin{array}{c}\text { Autor e } \\
\text { ano }\end{array}$ & $\begin{array}{c}\text { Recurso } \\
\text { tecnológico }\end{array}$ & Estratégia & Objetivo & $\begin{array}{l}\text { Tipo de } \\
\text { estudo }\end{array}$ & $\begin{array}{l}\text { Tempo de } \\
\text { estudo }\end{array}$ & $\begin{array}{l}\text { Número de } \\
\text { pessoas e } \\
\text { controle }\end{array}$ & Resultados \\
\hline $\begin{array}{l}\text { Miller et al. } \\
\text { (2018) }\end{array}$ & $\begin{array}{l}\text { mPATH-CRC } \\
\text { (Tecnologia móvel } \\
\text { do paciente para } \\
\text { a saúde - CRC) }\end{array}$ & $\begin{array}{l}\text { Aplicativo para iPad } \\
\text { que permite aos } \\
\text { pacientes solicitar } \\
\text { testes de triagem e } \\
\text { envia mensagens } \\
\text { eletrônicas de } \\
\text { acompanhamento e } \\
\text { suporte. }\end{array}$ & $\begin{array}{l}\text { Informar os } \\
\text { pacientes sobre } \\
\text { a necessidade de } \\
\text { triagem, ajudando- } \\
\text { os a tomar uma } \\
\text { decisão. }\end{array}$ & ECR & $\begin{array}{l}24 \\
\text { semanas }\end{array}$ & $\begin{array}{l}450 \\
(223 / 227)\end{array}$ & $\begin{array}{l}\text { Eficaz no aumento } \\
\text { da taxa de rastreio } \\
\text { para CCR. }\end{array}$ \\
\hline $\begin{array}{l}\text { Coronado } \\
\text { et al. } \\
\text { (2018) }\end{array}$ & $\begin{array}{l}\text { Programa de } \\
\text { extensão de teste } \\
\text { imunoquímico } \\
\text { fecal (FIT) } \\
\text { embutido no } \\
\text { registro eletrônico } \\
\text { de saúde (EHR) }\end{array}$ & $\begin{array}{l}\text { Envio de carta } \\
\text { introdutória, teste } \\
\text { FIT e carta-lembrete } \\
\text { encaminha dos pelo } \\
\text { correio. }\end{array}$ & $\begin{array}{l}\text { Identificar aqueles } \\
\text { que deveriam ser } \\
\text { submeti dos à } \\
\text { triagem do CCR. }\end{array}$ & ECR & 12 meses & $\begin{array}{l}41.193 \\
(21.134 / \\
20.059)\end{array}$ & $\begin{array}{l}\text { Aumento } \\
\text { significativo das } \\
\text { taxas de conclusão } \\
\text { de FIT e das } \\
\text { taxas de qualquer } \\
\text { rastreamento de } \\
\text { CCR. }\end{array}$ \\
\hline $\begin{array}{l}\text { Steffen et } \\
\text { al. (2015) }\end{array}$ & $\begin{array}{l}\text { Tele } \\
\text { CARE }\end{array}$ & $\begin{array}{l}\text { Envio de folheto } \\
\text { educacional e } \\
\text { recursos visuais } \\
\text { personalizados para o } \\
\text { rastreamento do CCR, } \\
\text { além de telefonemas } \\
\text { para avaliação do } \\
\text { risco de câncer. }\end{array}$ & $\begin{array}{l}\text { Aumentar a } \\
\text { captação de } \\
\text { colonoscopia } \\
\text { entre parentes de } \\
\text { pacientes com } \\
\text { câncer colorretal } \\
\text { que foram } \\
\text { considerados de } \\
\text { risco aumentado. }\end{array}$ & ECR & 15 meses & $\begin{array}{l}481 \\
(232 / 249)\end{array}$ & $\begin{array}{l}\text { Aumento do número } \\
\text { de triagem de } \\
\text { colonoscopia, porém } \\
\text { o custo ainda é uma } \\
\text { barreira. }\end{array}$ \\
\hline $\begin{array}{l}\text { Hagoel et } \\
\text { al. (2016) }\end{array}$ & $\begin{array}{l}\text { Mensagens de } \\
\text { texto }\end{array}$ & $\begin{array}{l}\text { Envio de carta-convite } \\
\text { mensagens de texto e } \\
\text { FOBT pelo correio. }\end{array}$ & $\begin{array}{l}\text { Informar sobre } \\
\text { a importância } \\
\text { de se realizar a } \\
\text { triagem para CR, } \\
\text { aumentando a taxa } \\
\text { de rastreio. }\end{array}$ & ECR & 6 meses & $\begin{array}{l}48.091 \\
(38.489 / \\
9.602)\end{array}$ & $\begin{array}{l}\text { Modesta mente } \\
\text { eficaz no rastreio } \\
\text { do CCR. }\end{array}$ \\
\hline $\begin{array}{l}\text { Mehta et } \\
\text { al. (2018) }\end{array}$ & $\begin{array}{l}\text { Mensagens } \\
\text { através do } \\
\text { registro eletrônico } \\
\text { de saúde (EHR) }\end{array}$ & $\begin{array}{l}\text { Envio de convites por } \\
\text { meio do EHR para } \\
\text { receber kits FIT. }\end{array}$ & $\begin{array}{l}\text { Alertar sobre a } \\
\text { importância da } \\
\text { pesquisa ativa } \\
\text { para CRC e enviar } \\
\text { do teste FIT para } \\
\text { realização da coleta } \\
\text { de material para } \\
\text { análise. }\end{array}$ & ECR & 3 meses & $\begin{array}{l}314 \\
(156 / 158)\end{array}$ & $\begin{array}{l}\text { Resultado mais } \\
\text { satisfatório no } \\
\text { grupo-controle que } \\
\text { recebeu por correio } \\
\text { que no grupo de } \\
\text { intervenção. }\end{array}$ \\
\hline $\begin{array}{l}\text { Brumbach } \\
\text { et al. } \\
\text { (2017) }\end{array}$ & $\begin{array}{l}\text { Tele-Cancer Risk } \\
\text { Assessment } \\
\text { and Evaluation } \\
\text { (TeleCARE) }\end{array}$ & $\begin{array}{l}\text { O grupo TeleCARE } \\
\text { recebeu } \\
\text { aconselhamento } \\
\text { sobre o CCR por } \\
\text { telefonema com um } \\
\text { especialista. }\end{array}$ & $\begin{array}{l}\text { Comunicar o risco } \\
\text { do CCR e mostrar } \\
\text { as vantagens da } \\
\text { colonoscopia, } \\
\text { visando aumentar } \\
\text { as motivações para } \\
\text { o rastreio. }\end{array}$ & ECR & 9 meses & $\begin{array}{l}496 \\
(247 / 249)\end{array}$ & $\begin{array}{l}\text { Aumento pequeno } \\
\text { no número de } \\
\text { rastreamento por } \\
\text { colonoscopia } \\
\text { nos usuários do } \\
\text { TeleCARE. }\end{array}$ \\
\hline
\end{tabular}

ECR: Ensaio clínico randomizado

Fonte: os autores (2020)

Nos estudos que abordavam o assunto triagem, portanto, dois utilizaram aplicativo e/ou programas eletrônicos (MEHTA et al., 2018; MILLER et al., 2018), um fez uso de carta e telefonema (STEFFEN et al., 2015); um fez uso de carta e programa eletrônico (CONORADO et al., 2018); um fez uso de mensagens de texto (HAGOEL et al., 2016); e um, de telefonema (BRUMBACH et al., 2017). Dentre os seis artigos, cinco relataram eficácia em seus programas (STEFFEN et al., 2015; HAGOEL et al., 2016; BRUMBACH et al., 2017; MILLER et al., 2018; CONORADO et al., 2018), contudo, em dois deles, a eficácia apresentada foi moderada (HAGOEL et al., 2016; BRUMBACH et al., 2017). Por outro lado, um dos estudos não demonstrou eficácia em sua intervenção (MEHTA et al., 2018). Apenas um trabalho fez uso da telemedicina para apoio ao atendimento desses pacientes (BRUMBACH et al., 2017).

O aplicativo para iPad denominado Patient Technology for Healh - CRC (m-PATH-CRC), auxilia o paciente a tomar decisões sobre o rastreamento do CCR, fornecendo informações sobre a doença, além de enviar mensagens a fim de auxiliar a realização e a conclusão do teste, como, por exemplo, incentivar 
a preparação do intestino, no caso de uma colonoscopia. O programa criado dobrou a proporção de pacientes que concluíram a triagem do CCR (MILLER et al., 2018).

Um artigo utilizou ferramentas personalizadas para identificar os pacientes elegíveis para Triagem do CCR, permitindo a elaboração de listas de correspondências, contendo uma carta introdutória, um pacote de kits de teste imunoquímico fecal (FIT), incluindo instruções sobre como concluir o teste, e uma carta-lembrete. As clínicas onde foram realizadas a intervenção obtiveram taxas mais altas de rastreamento do CCR (CONORADO et al., 2018).

Mensagens de texto de celular foram enviadas no estudo de Hagoel et al. (2016), para o recrutamento de 48.091 participantes para a triagem. Concluiu-se que as mensagens de texto foram eficazes na promoção da triagem, além de identificar os indicadores de fatores de risco (HAGOEL et al., 2016).

Um dos estudos comparou grupos de participantes que foram contactados pela internet (portal EHR) ou pelo correio. A pesquisa incluía o envio de kits FIT, os quais os participantes escolheriam receber ou não. Foi demonstrado uma participação maior, quando a divulgação da triagem CCR foi enviada por correio (MEHTA et al., 2018).

A ferramenta TeleCare foi utilizada em dois estudos. 0 primeiro abordava sobre o uso da Telemedicina, usando o recurso, após os participantes receberem brochuras educacionais e auxílios visuais enviados pelo correio. Para o acompanhamento da pesquisa, cada paciente recebeu um telefonema de um especialista e cartas personalizadas com o plano de ação individual para a realização da colonoscopia. 0 artigo apresentou aumento das taxas de triagem, quando realizada intervenção por TeleCare (STEFFEN et al., 2015). No outro estudo, um especialista para aconselhamento sobre os riscos do câncer entrava em contato com os participantes por telefone, cartas de acompanhamento personalizadas e lembretes de cartões postais. Os participantes poderiam responder por correio, internet ou telefone. A pesquisa obteve resultados positivos, mas modestos, no uso do TeleCare (BRUMBACH et al., 2017).

\section{CONSIDERAÇÕES FINAIS}

Neste trabalho, foi encontrado a aplicação de recursos tecnológicos da saúde digital em duas grandes áreas para CCR: triagem de novos pacientes e acompanhamento de pacientes em tratamento ou pós-tratamento.

Não encontramos artigos sobre eLearning, treinamentos médicos ou gestão aplicada ao CCR. A telemedicina, que poderia auxiliar no apoio mais próximo dos médicos aos pacientes, foi utilizada apenas em quatro estudos.

Considerando a grande incidência do CCR e suas altas taxas de mortalidade, é fundamental a introdução das ferramentas tecnológicas no âmbito da telemedicina e telessaúde, a fim de proporcionar melhorias na vida dos pacientes. Através dessas tecnologias, pode-se alcançar populações cada vez maiores, em curtos períodos de tempo, atuando na prevenção, rastreio, tratamento, monitoração e reabilitação do paciente com câncer colorretal. Como exemplo, é possível citar o diagnóstico precoce por imagem e o auxílio a um diferente estilo de vida. Portanto, a avaliação dos benefícios da telemedicina e telessaúde na questão da prevenção do câncer é de extrema importância.

Os resultados deste estudo contribuem para demosntrar a escassez de pesquisas na literatura com maior nível de evidência que possam auxiliar no tratamento, acompanhamento e prevenção do CCR na população. Novos trabalhos, portanto, são desejáveis para contribuir no cuidados desses pacientes. 


\section{REFERÊNCIAS}

BAKKER, Chantal M Den et al. Electronic Health Program to Empower Patients in Returning to Normal Activities After Colorectal Surgical Procedures: mixed-methods process evaluation alongside a randomized controlled trial. Journal of Medical Internet Research, [S.L.], v. 21, n. 1, p. 10674-10674, 29 jan. 2019. JMIR Publications Inc.. http://dx.doi.org/10.2196/10674.

BEDNARSKI, B. K. et al. Randomized clinical trial of accelerated enhanced recovery after minimally invasive colorectal cancer surgery (RecoverMI trial). Bjs, [S.L.], v. 106, n. 10, p. 1311-1318, 19 jun. 2019.

BERKEL, Annefleur E. M. et al. The effects of prehabilitation versus usual care to reduce postoperative complications in high-risk patients with colorectal cancer or dysplasia scheduled for elective colorectal resection: study protocol of a randomized controlled trial: study protocol of a randomized controlled trial. Bmc Gastroenterology, [S.L.], v. 18, n. 1, 21 fev. 2018. Springer Science and Business Media LLC. http:// dx.doi.org/10.1186/s12876-018-0754-6. Disponível em: https://pubmed.ncbi.nlm.nih.gov/29466955/?from_ term $=\% 22$ colorectal+cancer\%22+\&from_filter=pubt.clinicaltrial\&from_filter=ds1.y_5\&from_pos=2. Acesso em: 26 abr. 2020.

BRASIL. ESTIMATIVA 2020 INCIDÊNCIA DE CANCER NO BRASIL: incidência de câncer no brasil. Rio de Janeiro: Fox Print, v. 1, n. 1, 2019. Disponível em: https://www.saude.gov.br/images/pdf/2020/fevereiro/21/ estimativa-2020-incidencia-de-cancer-no-brasil.pdf. Acesso em: 26 abr. 2020.

BRUMBACH, Barbara H. et al. Intervention Mediators in a Randomized Controlled Trial to Increase Colonoscopy Uptake Among Individuals at Increased Risk of Familial Colorectal Cancer. Annals Of Behavioral Medicine, [S.L.], v. 51, n. 5, p. 694-706, 24 fev. 2017. Oxford University Press (OUP). http:// dx.doi.org/10.1007/s12160-017-9893-1.

CONSELHO FEDERAL DE MEDICINA. Resolução CFM n 2.228/2019 de 06 de fevereiro de 2019. Define e disciplina a telemedicina como forma de prestação de serviços médicos mediados por tecnologias. Entidades de Fiscalização do Exercício das Profissões Liberais/Conselho Federal de Medicina, Brasília, 2019. Disponível em: https://portal.cfm.org.br/images/PDF/resolucao222718.pdf. Acesso em: 11 ago. 2020.

CORONADO, Gloria D. et al. Effectiveness of a Mailed Colorectal Cancer Screening Outreach Program in Community Health Clinics. Jama Internal Medicine, [S.L.], v. 178, n. 9, p. 1174-1174, 1 set. 2018. American Medical Association (AMA). http://dx.doi.org/10.1001/jamainternmed.2018.3629.

ELDEIB, Hend K. et al. The Effect of Telephone-Based Follow-Up on Adherence, Efficacy, and Toxicity of Oral Capecitabine-Based Chemotherapy. Telemedicine And E-Health, [S.L.], v. 25, n. 6, p. 462-470, jun. 2019. Mary Ann Liebert Inc. http://dx.doi.org/10.1089/tmj.2018.0077.

GOLSTEIJN, Rianne Henrica Johanna et al. Short-term efficacy of a computer-tailored physical activity intervention for prostate and colorectal cancer patients and survivors: a randomized controlled trial. International Journal of Behavioral Nutrition And Physical Activity, [S.L.], v. 15, n. 1, p. 106-106, 30 out. 2018. Springer Science and Business Media LLC. http://dx.doi.org/10.1186/s12966-018-0734-9.

HAGOEL, Lea et al. Harnessing the Question-Behavior Effect to Enhance Colorectal Cancer Screening in an mHealth Experiment. American Journal of Public Health, [S.L.], v. 106, n. 11, p. 1998-2004, nov. 2016. American Public Health Association. http://dx.doi.org/10.2105/ajph.2016.303364. 
HAN, Jeong Yeob et al. How Cancer Patients Use and Benefit from an Interactive Cancer Communication System. Journal of Health Communication, [S.L.], v. 22, n. 10, p. 792-799, 18 set. 2017. Informa UK Limited. http://dx.doi.org/10.1080/10810730.2017.1360413. Disponível em: https://pubmed.ncbi.nlm.nih. gov/28922091/?from_term=\%22cancer\%22+and+\%22telemedicine\%22\&from_filter=pubt.clinicaltrial\&from_ filter=ds1.y_5\&from_pos=1. Acesso em: 26 abr. 2020.

HOUT, Anja van Der et al. Role of eHealth application Oncokompas in supporting self-management of symptoms and health-related quality of life in cancer survivors: a randomised, controlled trial. The Lancet Oncology, [S.L.], v. 21, n. 1, p. 80-94, jan. 2020.

MAYER, Deborah K. et al. SurvivorCHESS to increase physical activity in colon cancer survivors: can we get them moving?. Journal of Cancer Survivorship, [S.L.], v. 12, n. 1, p. 82-94, 9 out. 2017. Springer Science and Business Media LLC. http://dx.doi.org/10.1007/s11764-017-0647-7.

MEHTA, Shivan J. et al. A Randomized Controlled Trial of Opt-in Versus Opt-Out Colorectal Cancer Screening Outreach. American Journal of Gastroenterology, [S.L.], v. 113, n. 12, p. 1848-1854, dez. 2018. Ovid Technologies (Wolters Kluwer Health). http://dx.doi.org/10.1038/s41395-018-0151-3.

MILLER, David P. et al. Effect of a Digital Health Intervention on Receipt of Colorectal Cancer Screening in Vulnerable Patients. Annals of Internal Medicine, [S.L.], v. 168, n. 8, p. 550-550, 13 mar. 2018. American College of Physicians. http://dx.doi.org/10.7326/m17-2315.

OVERMAN, Michael $\mathrm{J}$ et al. Nivolumab in patients with metastatic DNA mismatch repair-deficient or microsatellite instability-high colorectal cancer (CheckMate 142): an open-label, multicentre, phase 2 study: an open-label, multicentre, phase 2 study. The Lancet Oncology, [S.L.], v. 18, n. 9, p. 1182-1191, set. 2017. Elsevier BV. http://dx.doi.org/10.1016/s1470-2045(17)30422-9. Disponível em: https://www. ncbi.nlm.nih.gov/pmc/articles/PMC6207072/. Acesso em: 26 abr. 2020.

PRESTON, Michael A.; GLOVER-COLLINS, Katherine; ROSS, Levi; PORTER, Austin; BURSAC, Zoran; WOODS, Delores; BURTON, Jacqueline; CROWELL, Karen; LARYEA, Jonathan; HENRY-TILLMAN, Ronda S.. Colorectal cancer screening in rural and poor-resourced communities. The American Journal of Surgery, [S.L.], v. 216, n. 2, p. 245-250, ago. 2018. Elsevier BV. http://dx.doi.org/10.1016/j.amjsurg.2017.08.004. Disponível em: https://pubmed.ncbi.nlm.nih.gov/28842164/?from_term=\%22colorectal+cancer\%22+\&from_filter=pubt. clinicaltrial\&from_filter=ds1.y_5\&from_pos=9. Acesso em: 26 abr. 2020.

STEFFEN, L. E. et al. Efficacy of a Telehealth Intervention on Colonoscopy Uptake When Cost Is a Barrier: the family care cluster randomized controlled trial. Cancer Epidemiology Biomarkers \& Prevention, [S.L.], v. 24, n. 9, p. 1311-1318, 22 jun. 2015. American Association for Cancer Research (AACR). http://dx.doi. org/10.1158/1055-9965.epi-15-0150.

WORLD HEALTH ORGANIZATION. eHealth [Internet]. 58th World Health Assembly; 16-25 May 2005; Geneva, Switzerland. Geneva: WHO; 2005 (Resolution WHA58.28). Disponível em: http://www.who.int/ healthacademy/media/WHA58-28-en.pdf?ua=1. Acesso: 19 fev. 2016. 